\title{
Measurement method of enterprise information asymmetry based on probability analysis
}

\author{
LIU Li ${ }^{1}$, JIANG Wen-bing ${ }^{2}$ \\ \{yueg00300@163.com¹,jwb00321@sina.com² \\ (1.Hunan University of Finance and Economics, Changsha 410205,China;
}

2.market director, guizhou east century science \& technology co., LTD.,Guiyang 550081, China)

\begin{abstract}
Aiming at the problem of low sensitivity in the traditional measurement method of enterprise information asymmetry degree, a probabilistic analysis based measurement method of enterprise information asymmetry degree is designed. By analyzing the causes and countermeasures of asymmetric information of enterprises, the proxy variables are determined, the coherence of proxy variables is calculated by probability analysis, and the cost of adverse selection of enterprises is decomposed by covariance decomposition method, which is combined with the coherence of proxy variables to calculate the degree of asymmetric information of enterprises. The experimental results show that compared with the traditional measurement methods, the method based on probability analysis is more sensitive and suitable for practical projects.
\end{abstract}

Keywords: Probability analysis; Information asymmetry; Measurement method;

\section{Introduction}

The information asymmetry theory is a kind of classical theory, which means that in the market economic activities, all kinds of people have different understandings of relevant information. Those who have enough information are often in a favorable position, while those who have little information are at a disadvantage ${ }^{[1]}$. According to the theory, the seller knows the goods better than the buyer in the market. The party with large amount of information benefits from the market by transmitting reliable information to the party with small amount of information; the buyer and the seller with small amount of information try to obtain information from the other party; market signal can make up for information asymmetry to some extent ${ }^{[2]}$.

In the enterprise, prone to two extremes, asymmetric information is a kind of adverse selection, due to the advance of information asymmetry, managers and other internal company personnel use may be bad for investors information favorable contract has been signed, while investors due to informational disadvantage in against their position, which makes the process 
of market transactions from the desire of the lack of information ${ }^{[3]}$. Influence the decision of investors and damage the interests of investors. The other is moral hazard, usually after the conclusion of the transaction contract, the work results depend on both the investment efforts of the agent and various objective factors not determined by the subjective will, and these two factors cannot completely distinguish or distinguish the cost of very high agent moral hazard problem $^{[4]}$.

For in the above case, adopt measure models or methods to measure the enterprise information asymmetry degree, according to the calculated result, the analysis of different degree of information asymmetry of adverse selection and moral hazard problems, from the angle of practical problems, through effective management means to reduce the company and the results of the capital market information asymmetry problem. Improving the governance mechanism of diversified companies, improving the relationship between the company and external investors, and achieving effective cooperation with the company's internal scientific management are the keys to give full play to the strategic advantages of diversified operations and ensure smooth financing channels.

Most of the previous methods for measuring the degree of information asymmetry of enterprises were based on the VAR model, and there was a problem of low sensitivity. Therefore, probabilistic analysis technology was adopted to design a method for measuring the degree of information asymmetry of enterprises based on probabilistic analysis, so as to solve the problems existing in the traditional methods.

\section{Measurement method of enterprise information asymmetry based on probability analysis}

\subsection{Identify the proxy variable for enterprise information asymmetry}

The agent variable of information asymmetry is the variable of information transaction, or the reason of information transaction, or the result of information transaction, which has certain causality. It is mainly the internal variables of the enterprise market, such as price change, bid-ask spread, turnover rate, internal ownership and enterprise growth ${ }^{[5]}$.

Before determining the proxy variable of enterprise information asymmetry, analyze the above variables and measure the value of the variables in measuring the degree of enterprise information asymmetry. According to the actual asymmetric information generation and response to determine the proxy variables.

Through the actual investigation of enterprises and markets, it is found that when the participants of enterprises and the market are faced with information asymmetry, they usually increase the sellers or decrease the buyers to avoid the losses caused by the exchanges with information traders. Therefore, the bid-ask spread is taken as one of the proxy variables. Due 
to the serious social informatization at the present stage, the information transactions in enterprises are becoming larger and larger, and the volume of transactions involved is also becoming larger and larger. If there is no information transaction in the market, the enterprise holds a diversified portfolio and trades according to a certain proportion of the number of shares outstanding by other individuals; If a company believes that it has private information about the stock market, the information trader's trade will disrupt the stock market. Thus, turnover is also one of the proxy variables; at the same time, the internal personnel of the growth enterprise have an advantage over the general external personnel in understanding the investment opportunities of the enterprise and the future cash flow generated by the existing assets of the enterprise. Therefore, the growth of enterprises and the investment opportunities of enterprises can be used as the proxy variables of information asymmetry.

From the perspective of the value of enterprise information itself, the price of information itself, the number of information transactions, the demand of enterprises or markets for information and the information provided by enterprises or markets are regarded as the proxy variables to measure the information asymmetry of enterprises. The coherence between enterprise information's own variables is shown in Figure 1.

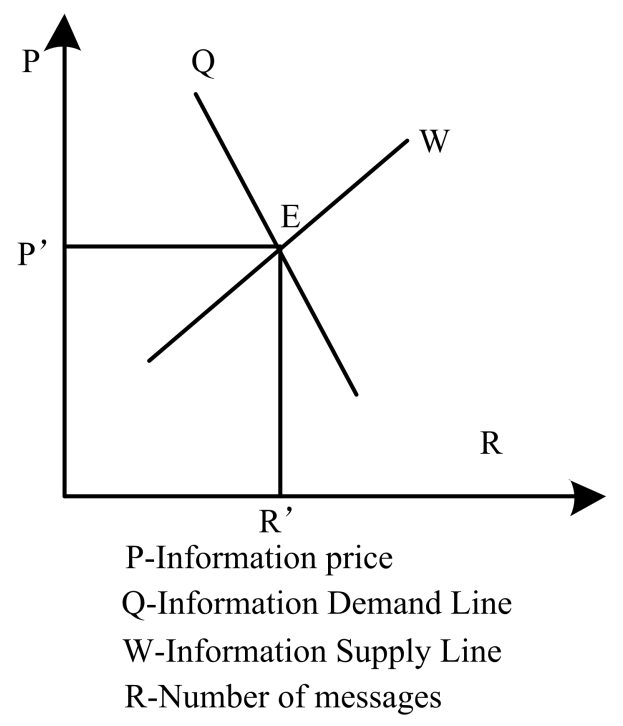

Fig. 1. Correlation between enterprise information's own variables

Probability analysis is used to calculate the probability distribution of the above basic variables, and the coherence of each variable to the measurement of enterprise information asymmetry degree is calculated. 


\subsection{The coherence calculation of proxy variables based on probability analysis}

In the calculation of the coherence of proxy variables, multiple variable parameters are involved. Before the coherence calculation, the data of the above variables are randomly sampled to obtain the characteristic values of sample mean, variance, mathematical expectation, skewness coefficient and kurtosis coefficient, which to some extent reflect the distribution characteristics of proxy variables ${ }^{[6]}$. The random sampling process is shown in Figure 2.

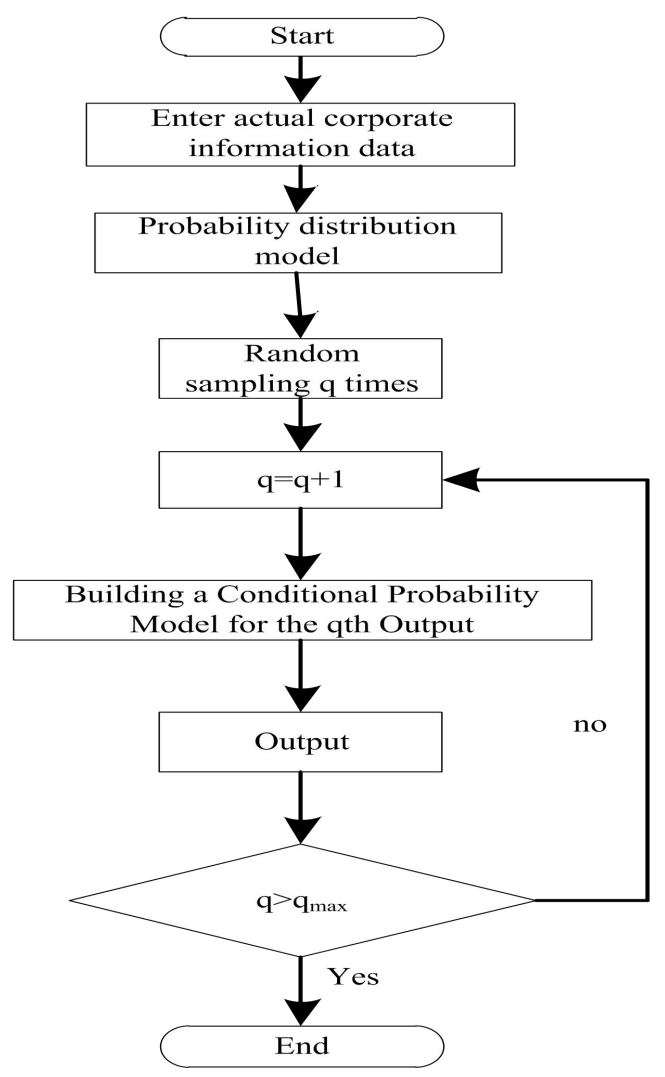

Fig. 2. Flow chart of random sampling of proxy data

Using the sampled data, the mathematical expectation, variance, skewness coefficient and kurtosis coefficient are calculated to reflect the distribution characteristics of the proxy variables. The calculation formula is as follows:

$$
e_{i}^{2}=\frac{\sum(u-\bar{u})}{n}
$$




$$
\begin{gathered}
E(i)=\sum_{n=1}^{N} u_{n} p_{i} \\
h_{i}=E\left[\left(\frac{u_{n} p_{i}}{e^{3}}\right)\right] \\
g_{i}=\frac{E\left[\left(h_{i}-e\right)^{4}\right]}{E\left(e_{i}^{2}\right)^{2}}
\end{gathered}
$$

In the formula, $e_{i}^{2}$ represents the variance of the $i$ th variable, $\bar{u}$ represents the mean of sample data, $u$ represents the sample data, $n$ represents the number of sample data, $E(i)$ represents the mathematical expectation of the $i$ th variable, $p_{i}$ represents the probability distribution of the $i$ th variable, $h_{i}$ represents the skewness coefficient of the $i$ th variable, and $g_{i}$ represents the kurtosis coefficient ${ }^{[7]}$. The above eigenvalues can only roughly reflect the characteristics of the distribution. In order to better calculate the coherence of the proxy variables, the distribution function of the proxy variables is obtained by histogram, and a probability distribution model is fitted by combining the eigenvalues. The coherence of the proxy variables is calculated by using the fitted probability distribution model.

The obtained eigenvalues are arranged in order of size, and the sequence of obtained variables is called empirical distribution ${ }^{[8]}$, Then these values are assigned to a series of intervals, where the interval division is estimated by Struges formula according to the sample size Q:

$$
W=1+3.3 \lg Q
$$

After the upper limit $a$ and lower limit $b$ of the data are known, and the number of intervals $W$ is selected, the interval interval can be calculated:

$$
\eta=\frac{a-b}{W} \text { (6) }
$$

Sometimes the value of $\mathrm{Q}$ is not necessarily strict in order to make the interval interval 


\section{$\eta$ a neat data.}

The calculation results can be adjusted properly. After the interval is divided, the median value of each interval is taken as the representative value of all data in the interval, the frequency, frequency and cumulative frequency of each interval are calculated, and the histogram is drawn ${ }^{[9]}$. The relation between frequency and cumulative frequency histogram is equivalent to the relation between probability density function and probability distribution function.

The normal distribution probability density curve is used to fit the above probability distribution model ${ }^{[10]}$. The probability density function is as follows:

$$
\beta_{i}(x)=\frac{1}{e \sqrt{2 \pi}} \exp \left[-\frac{1}{2}\left(\frac{x-\mu}{e}\right)^{2}\right]
$$

In the formula, $x$ and $\mu$ represent the distribution parameters. It is assumed that the sample data of the proxy variable is $u_{1}, u_{2}, \ldots, u_{n}$, and the probability distribution model after fitting is $F_{i}(u)$. The normal distribution is shown in Figure 3.

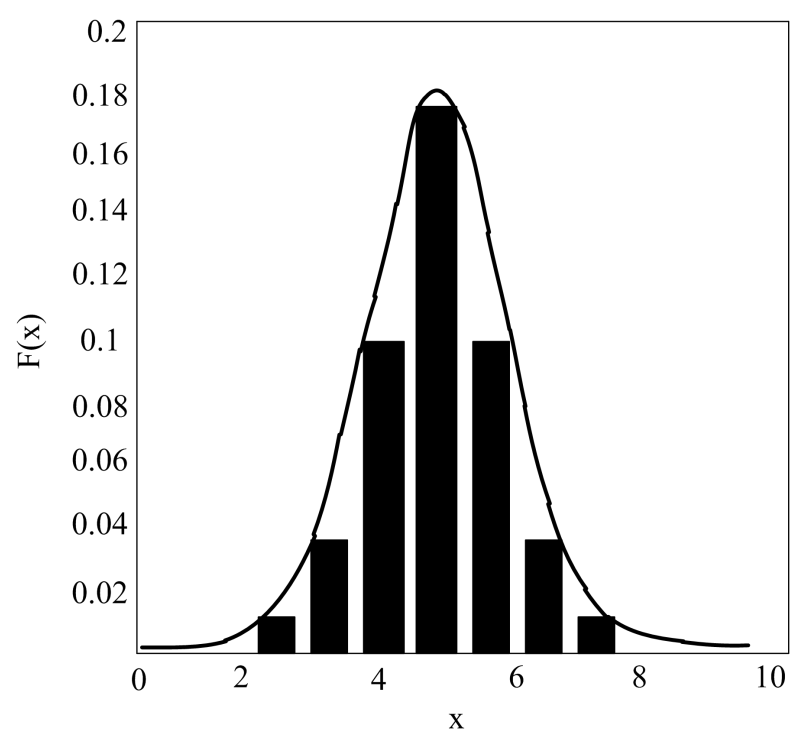

Fig. 3. Normal distribution

According to the sample interval and the size of the sample data, it is divided into groups 
of data without intersection, then the coherence of the proxy variable is as follows:

$$
\gamma_{i}=\sum_{z=1}^{Z} \frac{\left(\beta_{i}-n u_{z}\right)}{n u_{z}}
$$

In the formula, $u_{z}$ represents the sample data of the $z$ th interval. Through the above process, the coherence of proxy variables in the measurement of enterprise information asymmetry is calculated, which is combined with the decomposed adverse selection cost to calculate the degree of enterprise information asymmetry.

\subsection{Decompose the adverse selection cost}

According to the microstructure theory, the asset price of an enterprise is affected by instruction processing cost, survival cost and adverse selection cost, among which the adverse selection cost reflects the degree of information asymmetry of the market. By decomposing short-term price changes, various costs are separated out. If asymmetric information is the only factor that affects the spread between bid and offer prices, the transaction will reflect the information transmitted by the exchange. A buy with a buy quote results in a sustained increase in the buy and sell quote, reflecting the information transmitted by the buy exchange. If liquidity costs are the source of displacement in the spread, then the quote adjustment reflects an inventory balancing transaction. After the buy quote is sold, the buy quote does not decline, but the price change is not permanent. Covariance method was used to decompose bid-ask spread into transaction cost, revenue cost, expense cost and spread.

The method of covariance decomposition is used to decompose the adverse selection cost. The covariance matrix of the actual factors in the cost of adverse selection is decomposed to achieve the purpose of decompressing the cost of adverse selection. The covariance is expressed as:

$$
s(n)=\sum_{i=1}^{N} \phi_{i} v_{i}
$$

In the formula, $\phi_{i}$ represents the non-negative eigenvalue, $v_{i}$ represents the corresponding eigenvector, and $i$ represents the number of costs after the inverse selection cost decomposition.

The decomposition of adverse selection cost is realized by the method of differential solution, and the parameters of transaction cost, revenue cost and expenditure cost after decomposition are used in the calculation of subsequent information asymmetry degree. 


\subsection{Calculate the degree of information asymmetry}

Assuming that in a typical enterprise, there are many suppliers and demanders, both of which have certain information, the final transaction price is:

$$
d=\underline{d}+\chi\|\bar{d}-\underline{d}\|(10)
$$

In the formula, $\underline{d}$ represents the lowest transaction price acceptable to the supplier, $\bar{d}$ represents the highest transaction price willing to pay by the demand side, and $\chi(0 \leq \chi \leq 1)$ represents the degree of information available to the supplier in the pricing process. Therefore, $\chi\|\bar{d}-\underline{d}\|$ reflects the surplus captured by the supplier in the process of reaching the transaction price.

Next, the corresponding formula is used to show the degree of information acquired in the pricing process of the supply side and the demand side. Describe the "fair" transaction price of $r(x)$ under the given conditions of individual basic characteristics ii, and it always meets: $\underline{d} \leq r(x) \leq \bar{d}$, so $(\bar{d}-r(x))$ represents the expected surplus of the demand side in the transaction price reaching process; $(r(x)-\underline{d})$ represents the expected surplus of the supplier. Which side can "grab" more of the surplus will depend on the level of information they possess and the bargaining power based on it. With these remaining definitions, the formula is reformulated as:

$$
d=r(x)+[\underline{d}-r(x)]+\chi[\bar{d}-r(x)]-\chi[\underline{d}-r(x)]
$$

Formula (11) shows that the supplier can increase the transaction price by taking part of the surplus expected by the demander, and the surplus size is $\chi[\bar{d}-r(x)] ;$ In the same way, the demand side can reduce the transaction price by taking part of the surplus of the supply side, and the surplus size is $(1-\chi)[r(x)-\underline{d}]$. The surplus captured by the supplier depends on the degree of information possessed by the supplier $\chi$ and the total expected surplus of the demander $\bar{d}-r(x)$. Similarly, the surplus obtained by the demand side 
depends on the information level of the patient $(1-\chi)$ and the total expected remaining $r(x)-\underline{d}$ of the supply side, while the demand side can also lower the transaction price through the information level it has acquired.Therefore, in formula 11, it is mainly composed of three parts. The first part $r(x)$ represents the "fair" transaction price given individual characteristics, which is called the benchmark price. The second part $\chi[\bar{d}-r(x)]$ reflects the surplus captured by the supplier through the degree of information. The third part $(1-\chi)[r(x)-\underline{d}]$ is the surplus obtained by the demand side through the degree of information. Net surplus $F x=\chi[\bar{d}-r(x)]-(1-\chi)[r(x)-\underline{d}]$ can be used to describe the degree of information asymmetry in the formation of transaction prices.

The above process is an ideal state, measuring the degree of the asymmetry of the structure of the enterprise in the process of actual measurement, there are some interference is proxy variables and adverse selection costs, from the above process, the interference by a probability distribution, has the characteristics of normal distribution, therefore, derive the probability density function of complex interference item $\xi_{i}$ as follows:

$$
F\left(\xi_{i}\right)=\frac{\exp \left(\gamma_{i}\right)}{e_{i}}+\frac{\exp \left(v_{i}\right)}{\phi_{i}}
$$

For the measurement of information asymmetry degree of enterprises, it is mainly measured by the surplus obtained by the information degree mastered by the supply side and the demand side. Therefore, the net surplus in the bargaining process is calculated as follows:

$$
F x=\xi_{i}[(\chi \bar{d})-r(x)+(1-\chi) \underline{d}]
$$

The net surplus obtained through the above process is the degree of enterprise information asymmetry, so the measurement method of enterprise information asymmetry based on probability analysis has been designed.

\section{Simulation experiment and analysis}

\subsection{Set experimental constraints}

Because it is difficult to obtain real enterprise information, MATLAB software is used to 
simulate the experimental data. Prior to this, in order to prevent external factors from interfering with the experimental results, experimental constraints were formulated to ensure the effectiveness of the experiment.

According to the hypothesis of "information diversification", the level of diversification has a negative effect on the degree of information asymmetry, that is, the higher the level of diversification, the lower the degree of internal and external information asymmetry. The correlation between the operating departments of diversified enterprises and the estimation bias is consistent. The higher the level of non-correlated diversification is, the lower the degree of internal and external information asymmetry is. Therefore, constraint 1 is proposed. Information diversification has no influence on the degree of internal and external information asymmetry, and internal and external information is unified.

According to the hypothesis of "information transparency", the level of information transparency will have a positive effect on the degree of information asymmetry, that is, the higher the level of information transparency, the higher the degree of internal and external information asymmetry should be. However, when there is a high degree of correlation between different departments of an enterprise, the accuracy of reflecting the operating conditions of the department through the overall cash flow will also affect the transparency of information. Therefore, constraint condition 2 is put forward. Information transparency has no influence on the degree of internal and external information asymmetry of enterprises, and enterprise information transparency is unified.

\subsection{Sensitivity test results and analysis}

Under the above constraints, we use MATLAB software to extract the sample information of enterprises, and use the design budget method and the traditional budget method to measure the sensitivity of enterprise information asymmetry. The experimental results are as follows: 


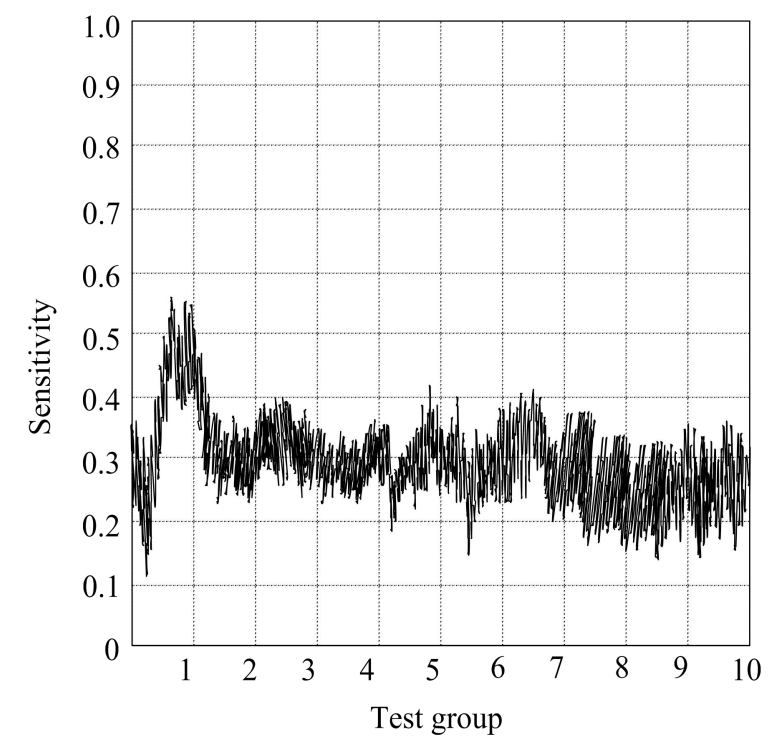

(a) Experimental results of traditional measurement methods

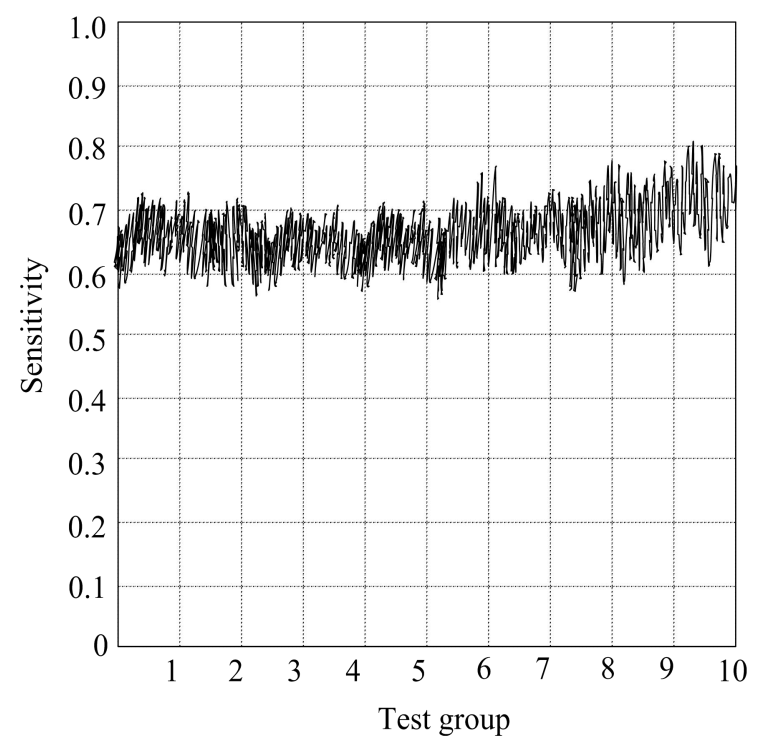

(b) Designed measurement method experimental results

Fig. 4. Experimental results of different measurement methods

According to the results in the observation figure, as shown in figure a, the sensitivity obtained by the traditional measurement method is between 0.2 and 0.6 in the early stage, and 
basically stable between 0.2 and 0.4 in the later stage. As shown in figure b, the sensitivity obtained by using the designed measurement method is relatively stable, always between 0.6 and 0.8. Compared with the two, the designed measurement method is more sensitive than the traditional one, indicating that the designed measurement method based on probability analysis is better than the traditional one.

\section{Conclusion}

The degree of information asymmetry of an enterprise has an important impact on the management and development of an enterprise. Traditional measurement methods cannot meet the current measurement of enterprise information asymmetry degree. Therefore, a probabilistic analysis based measurement method of enterprise information asymmetry degree is designed. Through the design comparison experiment, it is proved that the design measurement method solves the problems existing in the traditional measurement method, which is of great significance for the future development of enterprises.

\section{Reference}

[1] Yonghong, M., Yanrui, L.: A multi-game model of cooperative enterprise innovation strategy based on incomplete information. Journal of Harbin Engineering University. pp.1656-1661 (2019)

[2] Hongzhu, C., Yanqing, Q., Kang, W., et al: Calculation Strategy and Empirical Study on Labor Cost for Delivering Community-based Integrated Pension Service and Medical Service. Chinese General Practice. pp.1915-1921+1926 (2019)

[3] Peng, L., Yan, X., Dege, L.: Analysis on China's Degree of Dependence on Foreign Trade in the View of Global Value Chains. Chinese Journal of Management Science. pp.68-77 (2019)

[4] Kaihua, Y., Shuijun, P., Yuan Y.: Estimation and Decomposition of China's Interregional Trade Cost from the Perspective of Value Added Trade. Statistical Research. pp.63-75 (2019)

[5] Jiaqiong, W., Jia, Q.: Industrial Agglomeration of High Growth Enterprises in China's High-tech Zones. Journal of Beijing Jiaotong University (Social Sciences Edition). pp.41-48 (2018)

[6] Fanbin, K., Qiaoling, G., Dan, P.: Evaluation on Overfertilization and Its Spatial-Temporal Difference about Major Grain Crops in China. Economic Geography. pp.201-210+240 (2018)

[7] Rongwei, N., Nai-kang, G.: The Empirical Study on Information Asymmetry and Liquidity Commonality. Journal of Shanxi Finance and Economics University. pp.45-60 (2018)

[8] Changhong, L., Jiaqi, L., Wengting, J., et al. Family Control and Enterprise Innovation Investment based on the Moderating of Information Transparency. Science \& Technology Progress and Policy. pp.106-112 (2018)

[9] Jingyu, Y., Dongdong, L.: Emission Trading Auction Mechanism Design Based on Bilateral Abatement Cost Information Asymmetry. Chinese Journal of Management Science. pp.146-153 (2018)

[10] Yuqi, M., Ruipeng, H., Yanyun, Z.: The study of ownership, industry difference and exit mechanism 
of high-tech enterprises. Studies in Science of Science. pp.1642-1649 (2018) 